\title{
Distributed Dynamic Spectrum Allocation for Secondary Users in a Vertical Spectrum Sharing Scenario*
}

\author{
Behtash BABADI ${ }^{\dagger, \dagger, \dagger \dagger \dagger \mathrm{a})}$ and Vahid TAROKH ${ }^{\dagger \mathrm{b})}$, Nonmembers
}

\begin{abstract}
SUMMARY In this paper, we study the problem of distributed spectrum allocation under a vertical spectrum sharing scenario in a cognitive radio network. The secondary users share the spectrum licensed to the primary user by observing the activity statistics of the primary users, and regulate their transmission strategy in order to abide by the spectrum sharing etiquette. When the primary user is inactive in a subset of the available frequency bands, from the perspective of the secondary users the problem reduces to a distributed horizontal spectrum sharing. For a specific class of networks, the latter problem is addressed by the recently proposed GADIA algorithm [1]. In this paper, we present analytical and numerical results on the performance of the GADIA algorithm in conjunction with the abovementioned vertical spectrum sharing scenario. These results reveal nearoptimal performance guarantees for the overall vertical spectrum sharing scenario.

key words: dynamic spectrum allocation, cognitive radios, vertical spectrum sharing
\end{abstract}

\section{Introduction}

One of the main challenges in almost every communication network is the scarcity of spectrum. Exclusive spectrum access may become very hard to maintain in the future, thereby necessitating flexible spectrum usage with the goals of increasing the overall system efficiency and system concept flexibility and scalability. Cognitive radio systems have been proposed as a solution to these challenges, and have been the center of much attention as a new paradigm of network communication [6].

Most frequency bands up to $6 \mathrm{GHz}$ (and beyond) are allocated by the Federal Communications Commission (FCC). However, measurements show that much of this frequency at any time instance is not fully utilized, at various

\footnotetext{
Manuscript received September 10, 2011.

Manuscript revised December 16, 2011.

${ }^{\dagger}$ The authors are with the School of Engineering and Applied Sciences, Harvard University, Cambridge, MA 02138, USA.

${ }^{\dagger \dagger}$ The author is with the Department of Anesthesia, Critical Care and Pain Medicine, Massachusetts General Hospital, Boston, MA 02114, USA.

${ }^{\dagger \dagger}$ The author is with the Department of Brain and Cognitive Sciences, Massachusetts Institute of Technology, Cambridge, MA 02139, USA.

*This work has been presented in part at the 2012 IEEE Consumer Communications and Networking Conference, Jan. 14-17, Las Vegas, NV, USA [2]. This research is supported in part by ARO MURI grant number W911NF-07-1-0376. The views expressed in this paper are those of the authors alone and not of the sponsor.

a)E-mail: behtash@seas.harvard.edu

b) E-mail: vahid@ seas.harvard.edu

DOI: 10.1587/transcom.E95.B.1044
}

locations of interest. Thus, intelligent devices, such as $\operatorname{cog}$ nitive radios, may allow better use of the spectrum by sharing these unused resources.

The problem of spectrum sharing is thus an important yet challenging issue in wireless networking. Several papers in the literature focus on this problem from various perspectives (See, for example, [1], [5], [7], [11], [13], [14], [17], [18], and [22]. For a detailed literature review, see [1] and [12]). Spectrum sharing can be carried out in vertical or horizontal fashions. In the vertical spectrum sharing scenario, there exist pre-established priorities for all the involved systems. In particular, the secondary users need to control their transmission in order to prevent interference on the primary users. In the horizontal spectrum sharing scenario, there are no pre-established priorities for the users. Horizontal spectrum sharing can be done with or without coordination, and already exists in several systems. For example, systems operating in the ISM band share the spectrum horizontally without coordination. Examples of horizontal spectrum sharing with coordination are Wi-Fi devices.

Vertical spectrum sharing is a particularly important problem in cognitive radios. However, whenever a primary user is inactive, the secondary users need to perform horizontal spectrum sharing in order to maximize their sum-rate. This motivates us to study efficient and robust methods for horizontal spectrum sharing, as a sub-problem of vertical spectrum sharing.

FCC has recently released the band $3650-3700 \mathrm{MHz}$ for cognitive transmission [8]. Currently, fixed satellite services and federal government stations are transmitting in this band. Certain geographical areas around these transmitters are not allowed for secondary transmission. Otherwise, cognitive transmissions are allowed subject to $25 \mathrm{~W}$ and $1 \mathrm{~W}$ per $25 \mathrm{MHz}$ bandwidth for fixed and mobile stations, respectively. Other bands of interest are the TV Broadcast bands $(6 \mathrm{MHz}$ channels which correspond to the designated channels 2 to 69 in the VHF and UHF portions of the radio spectrum), which are denoted by TV White Spaces. Other existing devices in parts of this band are wireless microphones. The FCC has announced recently that secondary devices may operate in TV White Spaces based on certain rules to be published, in order to control their interference to the primary users. This has caused lots of interest in network solutions and consumer devices for these bands (See, for example, [10], [19] and [20]).

In this paper, we consider a cognitive network scenario where the secondary users observe the primary link activity, 
and regulate their transmission accordingly. That is, the secondary users horizontally share the portion of the spectrum which is unoccupied by the primary link. The Greedy Asynchronous Distributed Interference Avoidance (GADIA) algorithm [1] is considered as the horizontal spectrum sharing algorithm to be incorporated in the above-mentioned vertical spectrum sharing scenario. We will present analytical and numerical results on how the GADIA algorithm performs in the vertical spectrum sharing scenario, and how the overall vertical spectrum sharing scenario can improve the performance of the network. In particular, 1) we obtain analytical results on the temporal evolution of the interference experienced by the primary receiver, as well the Shannon capacity of the primary link, and 2) we study the dynamical behavior of the network utility of the secondary users in the vertical spectrum sharing scenario.

The outline of this paper follows next. In Sect. 2, we give an overview of the existing algorithms for horizontal spectrum sharing, including a discussion of the GADIA algorithm. The system model and the vertical spectrum sharing scenario are discussed in Sect. 3. The main results of the paper regarding the dynamics of various network quantities are presented in Sect. 4 followed a discussion in Sect. 5. The simulation results are presented in Sect. 6. Finally, we conclude the paper in Sect. 7. The proofs of the results of Sect. 4 are presented in the Appendices A and B.

\section{The GADIA Algorithm: An Overview}

Before talking about the overall vertical spectrum sharing scenario, we briefly overview the GADIA algorithm and other existing methods for horizontal spectrum sharing.

\subsection{Decentralized Horizontal Spectrum Sharing}

Due to the ever-growing usage of spectrum in the modern world, the scarcity of spectrum is one of the most important challenges in the wireless communication paradigm. Decentralized horizontal frequency allocation has provided a viable solution to this challenge, specially in many emerging communication networks (such as ad hoc wireless networks, wireless sensor networks, cognitive radios, etc.), where no central frequency allocation authority is naturally available. There are various proposed methods for decentralized (distributed) frequency allocation in different contexts (See, for example, [4], [5], [7], [11], [13], [14], [17], [18], [22], and [23]). These methods include methods based on graph coloring for cognitive networks, greedy interference avoidance techniques, Iterative Water-filling for Digital Subscriber Lines (DSL), game theoretic approaches to dynamic spectrum allocation and methods based on auction theory. These approaches may either excessively simplify the interference models, or may not be decentralized, or may require too much information exchange between autonomous nodes/clusters, or may suffer from all these shortcomings. Additionally, they may be too complex to implement. We will give a brief overview of some of these ap- proaches which are relevant to this work.

Etkin et al. [5] use pice-wise constant power allocation for optimal power spectral density (PSD) shaping across different users in the network, as a solution to distributed spectrum sharing. They provide a number of achievability and existence results in the context of non-cooperative and cooperative game theory for obtaining efficiency and fairness, as well as a punishment-based mechanism. Another result in the context of game theory is established by Huang et al. [7], in which each user in the network announces a price to the other users, so that they can adapt their PSD accordingly. Other game-theoretic methods based on potential games have been proposed in [13] and [14].

Another strand of work considers the problem of spectrum balancing (See, for example, [4] and [23]). The objective of spectrum balancing, which has its main application in DSL systems, is to maximize the throughput of each user by shaping its transmission PSD, satisfying certain power constraints. Yu et al. [23] propose the method of Iterative Water-filling in order to solve this problem. In the case of two users, they show the existence and conditions on the uniqueness of a Nash equilibrium point for their proposed iterative algorithm. The Iterative Water-filling algorithm has high complexity and the resulting Nash equilibrium point is not necessarily the optimal solution (See [5] for a discussion).

The GADIA algorithm proposed in [1] is a simple, lowcomplexity, robust and fully decentralized algorithm for horizontal spectrum sharing. In the GADIA algorithm, each node, having knowledge about the interference it experiences, chooses the frequency band with the least amount of interference from the other nodes. The GADIA algorithm requires neither any information exchange between autonomous devices, nor even any knowledge of the existence of other autonomous entities. In particular, it has been shown that the GADIA algorithm outperforms the Iterative Water-filling algorithm in wireless networks operating in the low signal to interference regime [1]. Before giving an overview of the GADIA algorithm, we will talk about the network model under which the algorithm operates.

\subsection{Network Model}

The canonical network model, under which the GADIA algorithm is defined, consists of a number of co-existing and hence interfering sets of network nodes, where each set may or may not have an internal structure [1]. Many communication networks of interest can be represented in this canonical form. We will discuss the class of Clustered Wireless Networks as an important example of the canonical network.

Consider a set of transceivers distributed in space such that they can be partitioned into a union of possibly overlapping clusters. Each cluster is equipped with a cluster-head. Such networks commonly arise in the context of cognitive radios and sensor networks. According to the recent FCC order on TV white spaces [8], a personal/portable device must either be under the control of 
a fixed device or a personal/portable device that employs geo-location, database access and spectrum sensing or employ geo-location/database access and spectrum sensing itself. Therefore, the assumption of a clustered wireless network, in which each cluster consists of a number of users is very reasonable in the context of cognitive networks sharing white spaces horizontally.

Another example is the homogeneous/heterogeneous clustered sensor networks for target tracking and localization (See, for example, [3]). In such networks, agile clusters of pressure, sonar and magnetic sensors are formed for target tracking purposes. Each cluster is equipped with a cluster-head, which communicates with the cluster members, gathers their data and reports to a fusion center. Often times a number of such clusters coexist in the same spacetime neighborhood, and hence spectrum sharing is very desired in order to increase the throughput of the underlying intra-cluster communication links.

More explicitly, the network consists of a collection of nodes, $c_{i}, i=1,2, \cdots, N$, which co-exist and interfere with each other. We assume that the interference between the nodes is reciprocal and the leakage interference is symmetric. In the clustered wireless network scenario, this can be achieved by assuming that at each moment there is at most one active transmitter in each cluster. Suppose that node $c_{i}$ transmits with a power $P_{i}$. The channel gain between nodes $c_{i}$ and $c_{j}$ is denoted by $h_{i j}$. We also attribute a self-gain of $h_{i i}$ to cluster $c_{i}$. Note that the channel gains and self-gains are conceptually defined over the canonical network, and may have different interpretations according to the realization of the canonical network. For instance, in the case of clustered wireless networks, the channel gain $h_{i j}$ can be interpreted as the wireless link gain between the two transmitting users in clusters $i$ and $j$. Similarly, a selfgain of $h_{i i}$ for cluster $c_{i}$ can be interpreted as the typical link gain between the cluster-head and cluster members of cluster $i$. Moreover, we assume that the accessible spectrum is divided into $r$ bands, $b_{1}, b_{2}, \cdots, b_{r}$. At time $t$, the $i$ th node is in state $s_{i}(t) \in\{1,2, \cdots, r\}$, corresponding to the index of the frequency band it is using for communication purposes. Finally, we assume that the rate of change of the spatial distributions of the nodes in the network is much less than the processing/transmission rate. We adopt this network model for the secondary users in the cognitive radio network under study.

Let $\alpha_{i j}:=h_{i j} / h_{i i}$ and $f\left(s_{i}, s_{j}\right)$ denote the symmetric leakage interference of the frequency band $s_{i}$ into $s_{j}$. Let $I_{c_{i}}^{k}(t)$ denote the interference experienced by $c_{i}$ caused by all the nodes in frequency band $b_{k}$, if $c_{i}$ was transmitting in band $b_{k}$, i.e.,

$$
I_{c_{i}}^{k}(t)=\sum_{\substack{j=1 \\ j \neq i}}^{N} P_{j} \alpha_{i j} f\left(k, s_{j}(t)\right) .
$$

The interference experienced by node $c_{i}$ at time $t$, given the state of the system being $\left\{s_{1}(t), s_{2}(t), \cdots, s_{N}(t)\right\}$, is given by

$$
I_{c_{i}}(t):=I_{c_{i}}^{s_{i}(t)}(t)=\sum_{\substack{j=1 \\ j \neq i}}^{N} P_{j} \alpha_{i j} f\left(s_{i}(t), s_{j}(t)\right)
$$

The network utility of the secondary users is their weighted aggregate interference and is defined as

$$
U_{s}(t):=-\sum_{i=1}^{N} P_{i} I_{c_{i}}(t) .
$$

This network utility is closely related to the sum-rate of the network and is discussed in detail in [1].

\subsection{The GADIA Algorithm}

In the GADIA algorithm, nodes scan all the frequency bands $b_{1}, \cdots, b_{r}$ in an asynchronous manner over time. Each node chooses the frequency band in which it experiences the least interference from other nodes. Let $t^{(1)}, t^{(2)}, t^{(3)}, \cdots$ be the points in time when the different nodes scan the spectrum in order to update their frequency band. Each node, say $c_{i}$, updates its frequency band at time $t^{(\ell)}$ according to the following rule:

$$
s_{i}(t)=\arg \min _{j} I_{c_{i}}^{j}\left(t^{(\ell)}\right)
$$

where $t \in\left(t^{(\ell)}, t^{(\ell+1)}\right]$. If the minimizer is not unique, $c_{i}$ randomly picks one such minimizer.

The performance of the GADIA algorithm is studied comprehensively in [1]. In particular, the convergence of the GADIA algorithm to a local minimum is proved. Furthermore, several performance bounds in [1] show that this local minimum can be very close to the optimal solution for a wide range of spatial node configurations. Another class of results in [1] is the dynamical analysis of the GADIA algorithm, which is more relevant to this paper. In particular, using methods from statistical physics, the dynamical behavior of the utility function is derived.

Let $\mathscr{P}\left(t ; s_{1}, s_{2}, \cdots, s_{N}\right)$ be the probability density of the nodes $\left(c_{1}, c_{2}, \cdots, c_{N}\right)$ being at the point $\left(s_{1}, s_{2}, \cdots, s_{N}\right)$ in the state-space $\{1,2, \cdots, r\}^{N}$ at time $t$. For any scalar function $f\left(s_{1}, s_{2}, \cdots, s_{N}\right)$ over the state-space, the ensemble average at time $t$ is defined as follows:

$$
\begin{aligned}
& \left\langle f\left(s_{1}, s_{2}, \cdots, s_{N}\right)\right\rangle:= \\
& \quad \sum_{\sigma_{1}=1}^{r} \sum_{\sigma_{2}=1}^{r} \cdots \sum_{\sigma_{N}=1}^{r} f\left(\sigma_{1}, \sigma_{2}, \cdots, \sigma_{N}\right) \mathscr{P}\left(t ; \sigma_{1}, \sigma_{2}, \cdots, \sigma_{N}\right) .
\end{aligned}
$$

Moreover, suppose that the network nodes update their frequency bands with a rate of $1 / \tau_{s}$. Finally, assume that the leakage function $f\left(s_{i}, s_{j}\right)$ is approximated by the Kronecker delta function $\delta\left(s_{i}, s_{j}\right)$. Then, the ensemble average of the network utility satisfies the following differential equation:

$$
\frac{d}{d t}\left\langle U_{s}(t)\right\rangle=-\frac{2}{\tau_{s}}\left(\left\langle U_{s}(t)\right\rangle-\left\langle U_{0}(t)\right\rangle\right),
$$

where 


$$
U_{0}(t):=-\lim _{\beta \rightarrow \infty} \sum_{\substack{k, l=1 \\ k \neq l}}^{N} P_{k} P_{l} \alpha_{k l} F(k, l, \beta ; t)
$$

with

$$
F(k, l, \beta ; t):=\frac{\exp \left(-\beta \sum_{j \neq k} P_{k} P_{j} \alpha_{k j} \delta\left(s_{l}(t), s_{j}(t)\right)\right)}{\sum_{m} \exp \left(-\beta \sum_{j \neq k} P_{k} P_{j} \alpha_{k j} \delta\left(m, s_{j}(t)\right)\right)}
$$

Moreover, it has been shown that the dynamics can be simplified to

$$
\frac{d}{d t}\left\langle U_{s}(t)\right\rangle=-\frac{2}{\tau_{s}}\left(\left\langle U_{s}(t)\right\rangle-\langle U(\infty)\rangle\right)
$$

near equilibrium, where $U(\infty)$ is independent of $t$. Equation (8) corresponds to exponential dynamics with time constant $\tau_{s}$.

\section{System Model and Problem Formulation}

Before defining the technical specifications of the vertical spectrum sharing scenario, we provide a motivational example. Consider an urban scenario where pressure, magnetic and video sensors are located on the streets for traffic control or target tracking. These sensors may form clusters for data fusion. Also, they can use the Police spectrum or TV white spaces for their ad-hoc communication purposes. In this case, the secondary users are the sensors in the network, and the primary user is the Police or TV receiver. The secondary users need to abide by certain spectrum policies in order to avoid interfering with the licensed primary users.

Next, we build a mathematical model for the vertical spectrum sharing scenario under study.

\subsection{System Model}

First, we overview the network model under which we formulate the spectrum sharing problem. We consider a pair of transmitter/receiver primary users located arbitrarily in space. Motivated by the FCC order [8], certain geographical areas around these transmitters are not allowed for secondary transmission. Figure 1 shows the system model. The primary transmitter and receiver are a distance $d$ apart from each other. $R_{\min }$ denotes the radius of the primary-exclusive region dictated by the FCC. $R_{0}$ denotes the radius below which the secondary users are not allowed to transmit while the primary link is active. Finally, $R_{\max }$ denotes the maximum radius below which the interfering secondary users are located.

\subsection{The Vertical Spectrum Sharing Scenario}

Suppose that the primary transmitter/receiver pair are located as in Fig. 1. Moreover, suppose that the primary transmitter uses the $i$ th frequency band according to a two-state symmetric Markov process with a transition rate of $1 / \tau_{p}$.

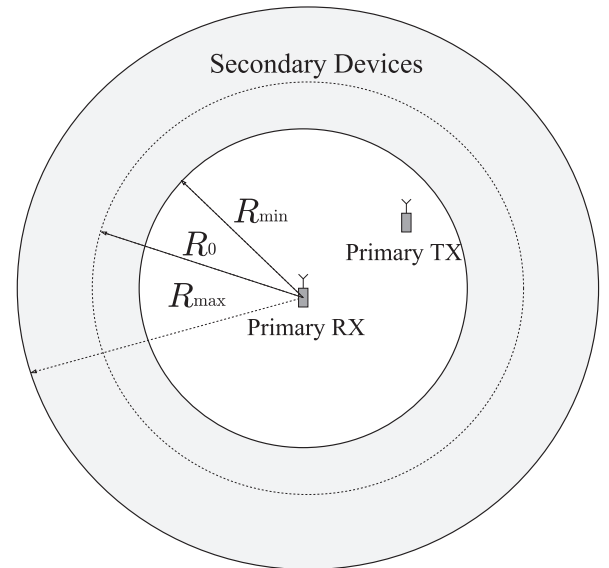

Fig. 1 System model

Let $A^{(i)}(t)$ denote the activity state of the primary link in the $i$ th frequency band $b_{i}$, i.e., $A^{(i)}(t)=0$ when the primary link does not transmit in band $b_{i}$, and $A^{(i)}(t)=1$ when the primary link transmits in band $b_{i}$, at time $t$. Therefore, the state of the primary link at time $t$ is given by $s(t):=\left(A^{(1)}(t), A^{(2)}(t), \cdots, A^{(r)}(t)\right) \in\{0,1\}^{r}$.

We assume that the secondary users form a network which lies in the canonical network category defined earlier. Finally, suppose that the secondary users sense the activity of the primary transmitter and scan the spectrum with rate $1 / \tau_{s}$. Then, the vertical spectrum sharing scenario is as follows:

Each secondary user senses the activity of the primary link in each of the available frequency bands, and conforms to the GADIA algorithm across the set of frequency bands $\left\{b_{i} \mid A^{(i)}(t)=0\right\}$ in order to share the spectrum with the other secondary users. All the secondary users within a distance of $R_{0}$ from the primary receiver must refrain from transmitting in any frequency band $b_{i}$ for which $A^{(i)}(t)=1$. The secondary users at a distance greater or equal to $R_{0}$ from the primary receiver do not need to regulate their transmission according to the primary link activity, and follow the GADIA algorithm for horizontal spectrum sharing across all the $r$ frequency bands.

Remark 1. Note that the distance $R_{0}$ is dictated by the FCC [8]. Assuming that the secondary users are equipped with geo-location devices, they can estimate their distance to the primary and hence abide by the vertical spectrum sharing etiquette.

Remark 2. The secondary users sense the activity of the primary using carrier sensing or beacon detection techniques. These techniques are discussed in detail in [8].

\section{Main Results}

Before presenting the main results of this paper, we introduce a few additional assumptions for the sake of analysis. First of all, we suppose that the channel model is governed by path loss with exponent $\eta$. This is a common simpli- 
fying assumption in the literature of cognitive radios (See, for example, [1], [15], and [21]). For simplicity, we assume that the spectrum spread is small enough so that the channel model is the same for all the $r$ available frequency bands. Moreover, we assume that the $N$ secondary users are distributed uniformly between the two discs of radius $R_{\min }$ and $R_{\max }$, with a density of $\Lambda=N / \pi\left(R_{\max }^{2}-R_{\min }^{2}\right)$. Finally, suppose that all the secondary users transmit with a power $\tilde{P}_{s}:=P_{s} / N$, and the primary transmitter transmits with a power $P_{p}$ in each of the $r$ frequency bands. Note that the parameter $P_{s}$ is employed as a scaled version of the secondary transmit power, which will be shown to be an convenient scaling in the limit of $N \rightarrow \infty$.

\subsection{Primary Dynamics}

Let $p_{\mathrm{fa}}$ and $p_{\mathrm{md}}$ denote the false alarm and miss detection probabilities for the secondary user $c_{j}$ in detecting the events $A^{(i)}(t)=0$ and $A^{(i)}(t)=1$, respectively, for all $i=1,2, \cdots, r$ and $j=1,2, \cdots, N$. Such errors occur due to the observation noise and statistics mismatch of the detection model [19]. Let $a_{j}^{(i)}(t)$ denote the activity state of the secondary user $c_{j}$ in the frequency band $b_{i}$, for $i=1,2, \cdots, r$ and $j=1,2, \cdots, N$. Finally, let $\mathcal{I} \subseteq\{1,2, \cdots, N\}$ be the indices corresponding to the secondary users within a distance $R_{0}$ from the primary receiver.

Suppose that the primary link state transitions occur at time instances $t_{1}, t_{2}, \cdots$. At time $t=t_{\ell}$, consider the primary link transition from state $s_{1}\left(t_{\ell}\right):=s\left(t_{\ell}^{-}\right)$to $s_{2}\left(t_{\ell}\right):=s\left(t_{\ell}^{+}\right)$. Assuming that the $r$ Markov processes governing the state transitions for each frequency band are independent, the probability that $s_{1}\left(t_{\ell}\right)$ and $s_{2}\left(t_{\ell}\right)$ differ in more than one frequency band is 0 . Let $i_{\ell}$ be the the frequency band in which the two state vectors $s_{1}\left(t_{\ell}\right)$ and $s_{2}\left(t_{\ell}\right)$ differ. Let $\mathcal{J}_{\ell}(t):=\left\{b_{i} \mid A^{(i)}\left(t_{\ell}^{+}\right)=1\right\}$, be the set of frequency bands in which the primary link is active after a transition at time $t_{\ell}$. We have two types of transition: type 1) $A^{\left(i_{\ell}\right)}\left(t_{\ell}^{-}\right)=0$ and $A^{\left(i_{\ell}\right)}\left(t_{\ell}^{+}\right)=1$, and type 2) $A^{\left(i_{\ell}\right)}\left(t_{\ell}^{-}\right)=1$ and $A^{\left(i_{\ell}\right)}\left(t_{\ell}^{+}\right)=0$. We first consider transitions of type 1 .

\section{Type 1 Transitions}

$A^{\left(i_{\ell}\right)}\left(t_{\ell}^{-}\right)=0$ and $A^{\left(i_{\ell}\right)}\left(t_{\ell}^{+}\right)=1$. In this case, the frequency band $i_{\ell}$ is not available to the secondary users within a distance of $R_{0}$ from the primary receiver.

Suppose that $\left|\mathcal{J}_{\ell}(t)\right|:=r-m$ for some $0 \leqslant m \leqslant r-1$. Then, at any time $t \geqslant t_{\ell}$, the probability that a secondary user $c_{i}$ with $i \in I$ chosen at random, which did not use the frequency band $b_{i_{\ell}}$ at $t<t_{\ell}$, starts using the frequency band $b_{i_{\ell}}$ is given by:

$$
\begin{aligned}
p_{e}:=p_{\mathrm{md}} & \sum_{l=0}^{m} \sum_{k=0}^{r-m-1} \frac{1}{l+k+1}\left(\begin{array}{c}
r-m-1 \\
k
\end{array}\right)\left(\begin{array}{c}
m \\
l
\end{array}\right) \\
& \times\left(1-p_{\mathrm{fa}}\right)^{l} p_{\mathrm{fa}}^{m-l} p_{\mathrm{md}}^{k}\left(1-p_{\mathrm{md}}\right)^{r-m-1-k} .
\end{aligned}
$$

In the time interval $\left[t_{\ell}, t_{\ell+1}\right]$, the dynamics of $a_{k}^{\left(i_{\ell}\right)}(t)$ is thus governed by the following Itô equation [9]:

$$
d a_{k}^{\left(i_{\ell}\right)}(t)=-a_{k}^{\left(i_{\ell}\right)}(t) d N_{k}+\left(1-a_{k}^{\left(i_{\ell}\right)}(t)\right) d N_{k}^{\prime}
$$

for $k=1,2, \cdots, N$, where $d N_{k}$ and $d N_{k}^{\prime}$ are two independent Poisson counters with rates $\left(1-p_{e}\right) / \tau_{s}$ and $p_{e} / \tau_{s}$, respectively [9]. Note that the Poisson counter $d N_{k}$ accounts for the successful sensing process of the secondary user $c_{k}$ (and thus refraining from transmission in band $b_{i_{\ell}}$ ), and $d N_{k}^{\prime}$ reflects erroneous sensing of the secondary user $c_{k}$, which results in transmitting in band $b_{i_{\ell}}$. Given that the secondary users carry out their sensing independently and homogenously, the interference experienced by the primary receiver in band $i_{\ell}$ at time $t \in\left[t_{\ell}, t_{\ell+1}\right]$ can be approximated by:

$$
I^{\left(i_{\ell}\right)}(t)=\frac{1}{r} I_{g}+\frac{n^{\left(i_{\ell}\right)}(t)}{N} I_{e}
$$

where

$$
\begin{aligned}
I_{g} & :=\frac{2 \pi \Lambda}{N(\eta-2)}\left(\frac{P_{s}}{R_{0}^{\eta-2}}-\frac{P_{s}}{R_{\max }^{\eta-2}}\right), \\
I_{e} & :=\frac{2 \pi \Lambda}{N(\eta-2)}\left(\frac{P_{s}}{R_{\min }^{\eta-2}}-\frac{P_{s}}{R_{0}^{\eta-2}}\right),
\end{aligned}
$$

and $n^{\left(i_{\ell}\right)}(t)$ is the number of active secondary users located within the radius $R_{0}$ from the primary receiver and transmitting in band $b_{i_{\ell}}$ at time $t$, i.e.,

$$
n^{\left(i_{\ell}\right)}(t):=\sum_{k \in \mathcal{I}} a_{k}^{\left(i_{\ell}\right)}(t) .
$$

Recall that the secondary users transmit with a power $\tilde{P}_{s}:=P_{s} / N$. We prefer to work with $P_{s}$ rather than $\tilde{P}_{s}$ for the clarity of the limiting cases discussed throughout this paper, and hence express all the underlying parameters in terms of the scaled power $P_{s}$. Note that $\frac{1}{r} I_{g}$ is the lowest possible interference on the primary receiver in band $b_{i_{\ell}}$, and corresponds to the case where none of the secondary users within the radius of $R_{0}$ are transmitting in band $b_{i_{\ell}}$, and $1 / r$ of the secondary users beyond the radius of $R_{0}$ are transmitting in band $b_{i_{\ell}}$ on average (due to the assumption of spatial homogeneity and symmetry of the $r$ frequency bands). That is, the primary experiences the minimum interference of $\frac{1}{r} I_{g}$ when all the secondary users are given genie knowledge of the activity state of the primary link in frequency band $b_{i_{\ell}}$ at time $t$. Moreover, $I_{e}$ corresponds to the excess interference caused by the secondary users which have not yet detected the activity state of the primary at time $t$, and are still transmitting in band $b_{i_{\ell}}$.

The dynamics of $n^{\left(i_{\ell}\right)}(t)$ is enough to obtain that of $I^{\left(i_{\ell}\right)}(t)$. Thus, we first study the dynamics of $n^{\left(i_{\ell}\right)}(t)$. Let $\mathcal{E}_{s}$ denote the ensemble averaging operator with respect to the Poisson counters $d N_{j}$ and $d N_{j}^{\prime}$, for $j=1,2, \cdots, N$. We then have the following theorem regarding the mean and variance of $n^{\left(i_{\ell}\right)}(t)$ :

Theorem 4.1: Suppose that at time $t=t_{\ell}$ the primary transmitter starts transmitting in frequency band $b_{i_{\ell}}$, and 
that $n_{\ell}:=n^{\left(i_{\ell}\right)}\left(t_{\ell}\right)$ secondary users within a distance of $R_{0}$ of the primary receiver are active in this band at time $t_{\ell}$. Let $p_{e}$ be the error probability that the secondary user $c_{i}$ starts transmitting in band $b_{i_{\ell}}$ at time $t \geqslant t_{\ell}$. Then, the mean and normalized variance of $n^{\left(i_{\ell}\right)}(t)$ at time $t_{\ell} \leqslant t \leqslant t_{\ell+1}$ are given by:

$$
\mathcal{E}_{s}\left\{n^{\left(i_{\ell}\right)}(t)\right\}=\left\{n_{\ell} e^{-\left(t-t_{\ell}\right) / \tau_{s}}+\alpha N p_{e}\left(1-e^{-\left(t-t_{\ell}\right) / \tau_{s}}\right)\right\}
$$

and

$$
\begin{aligned}
\sigma_{n^{\left(i_{\ell}\right)}(t)}^{2}:= & \frac{1}{N^{2}} \mathcal{E}_{s}\left\{n^{\left(i_{\ell}\right)}(t)^{2}-\mathcal{E}\left\{n^{\left(i_{\ell}\right)}(t)\right\}^{2}\right\} \\
= & \frac{\left(1-e^{-\left(t-t_{\ell}\right) / \tau_{s}}\right)}{N} \\
& \times\left\{\alpha_{\ell}\left(1-2 p_{e}\right) e^{-\left(t-t_{\ell}\right) / \tau_{s}}+\alpha p_{e}\left(1-p_{e}+p_{e} e^{-\left(t-t_{\ell}\right) / \tau_{s}}\right)\right\}
\end{aligned}
$$

where

$$
\alpha:=\frac{R_{0}^{2}-R_{\min }^{2}}{R_{\max }^{2}-R_{\min }^{2}}
$$

is the ratio of the number of secondary users within a distance $R_{0}$ of the primary receiver to the total number of secondary users.

The proof is given in Appendix A.

Remark 3. The evolution of $n^{\left(i_{\ell}\right)}(t)$ following a transition of $A^{\left(i_{\ell}\right)}\left(t_{\ell}^{-}\right)=1$ to $A^{\left(i_{\ell}\right)}\left(t_{\ell}^{+}\right)=0$ can be obtained similarly and is governed by the same Itô equation given in Eq. (10), with $d N_{k}$ replaced by $d N_{k}^{\prime}$, and $p_{\text {md }}$ replaced by $p_{\mathrm{fa}}$ in the definition of $p_{e}$ (and vice versa). The details are omitted for brevity.

From Theorem 4.1 and Eq. (11), the mean and normalized variance of the interference experienced by the primary received in band $b_{i_{\ell}}$ at time $t \in\left[t_{\ell}, t_{\ell+1}\right]$ are given by

$$
\mathcal{E}_{s}\left\{I^{\left(i_{\ell}\right)}(t)\right\}=\frac{1}{r} I_{g}+\frac{\mathcal{E}_{s}\left\{n^{\left(i_{\ell}\right)}(t)\right\}}{N} I_{e} \text {, and } \sigma_{I^{\left(i_{\ell}\right)}(t)}^{2}=I_{e}^{2} \sigma_{n^{\left(i_{\ell}\right)}(t)}^{2} .
$$

Note that the normalized variances of $n^{\left(i_{\ell}\right)}(t)$ and $I^{\left(i_{\ell}\right)}(t)$ vanish as $N \rightarrow \infty$. Hence, by an application of the Chebyshev's inequality [16], $n^{\left(i_{\ell}\right)}(t)$ and $I^{\left(i_{\ell}\right)}(t)$ converge to their mean almost surely, as $N \rightarrow \infty$. Thus, we have the following proposition:

Proposition 4.2: $\quad$ The normalized interference experienced by the primary receiver in band $b_{i_{\ell}}$ at time $t \in\left[t_{\ell}, t_{\ell+1}\right]$ converges to its mean value given by

$$
\mathcal{E}_{s}\left\{I^{\left(i_{\ell}\right)}(t)\right\}=\frac{1}{r} I_{g}+\left\{\alpha_{\ell} e^{-\left(t-t_{\ell}\right) / \tau_{s}}+\alpha p_{e}\left(1-e^{-\left(t-t_{\ell}\right) / \tau_{s}}\right)\right\} I_{e}
$$

almost surely as $N \rightarrow \infty$, where $\alpha_{\ell}:=n_{\ell} / N$. Furthermore, for $t$ large enough, the interference experienced by the primary in band $b_{i_{\rho}}$ gets arbitrarily close to the near-optimal value of $\frac{1}{r} I_{g}+\alpha_{\ell} p_{e} I_{e}$.

Remark 4. Note that when $p_{e}=0$, i.e., when the secondary users detect the activity state of the primary link without any error, the asymptotic value of the interference coincides with the optimal value of $\frac{1}{r} I_{g}$.

Type 2 Transitions

$A^{\left(i_{\ell}\right)}\left(t_{\ell}^{-}\right)=1$ and $A^{\left(i_{\ell}\right)}\left(t_{\ell}^{+}\right)=0$. In this case, both the interference and capacity of the primary receiver in band $b_{i_{\ell}}$ are equal to zero for $t \in\left[t_{\ell}, t_{\ell+1}\right]$.

Combining the above two cases, we can express the interference experienced by the primary receiver for all $t$. Let $t_{1}^{(j)}, t_{2}^{(j)}, \cdots$ show the time instances for which transitions of type 1 occur in band $b_{j}$. Similarly, let $t_{1}^{\prime(j)}, t_{2}^{\prime(j)}, \cdots$ denote the time instances corresponding to state transitions of type 2 in band $b_{j}$. Without loss of generality, suppose that

$$
t_{1}^{(j)}<t_{1}^{\prime(j)}<t_{2}^{(j)}<t_{2}^{\prime(j)}<t_{3}^{(j)}<t_{3}^{(j)}<\cdots
$$

Then, the asymptotic overall interference experienced by the primary receiver, $I(t)$, at time $t$ is given by

$$
I(t) \rightarrow \mathcal{E}_{s}\{I(t)\}=\tilde{I}_{g}(t)+\tilde{I}_{e}(t),
$$

where

$$
\begin{aligned}
& \tilde{I}_{g}(t):=\sum_{j=1}^{r} \frac{1}{r} A^{(j)}(t) I_{g}, \\
& \tilde{I}_{e}(t):=\sum_{j=1}^{r} \sum_{k: t_{k}^{(j)}, t^{\prime}{ }_{k}^{(j)} \leqslant t}\left\{\alpha_{k}^{(j)} e^{-\left(t-t_{k}^{(j)}\right) / \tau_{s}}+\alpha p_{e}\left(1-e^{-\left(t-t_{k}^{(j)}\right) / \tau_{s}}\right)\right\} I_{e} \\
& \times\left(u\left(t-t_{k}^{(j)}\right)-u\left(t-t_{k}^{(j)}\right)\right),
\end{aligned}
$$

and $\alpha_{k}^{(j)}:=n^{(j)}\left(t_{k}^{(j)}\right) / N$ is the portion of secondary users transmitting in band $b_{j}$ at time $t_{k}^{(j)}$. Note that $\tilde{I}_{g}(t)$ and $\tilde{I}_{e}(t)$ represent the normalized interference experienced by the primary receiver from all the secondary users beyond and within the radius $R_{0}$ at time $t$, respectively.

As an application of Eq. (14), one can consider the steady state behavior of the interference experienced by the primary receiver. We have the following proposition regarding the ensemble average of the interference experienced by the primary receiver (with respect to the temporal dynamics of the primary link activity):

Proposition 4.3: Suppose that the primary link activity in each of the $r$ frequency band is governed by an independent symmetric two-state Markov process with transition rate $1 / \tau_{p}$. Let $\mathcal{E}_{p}$ denote the ensemble average with respect to these set of independent Markov processes. Then, in the steady state we have:

$$
\mathcal{E}_{p}\{I(t)\} \rightarrow \mathcal{E}_{p}\left\{\mathcal{E}_{s}\{I(t)\}\right\}=\frac{1}{2} I_{g}+\frac{1}{2} \alpha\left(\frac{\xi(r) \tau_{s}+\gamma(r) p_{\mathrm{md}} \tau_{p}}{\tau_{s}+\tau_{p}}\right) I_{e},
$$

almost surely as $N \rightarrow \infty$, given $\tau_{s} \ll \tau_{p}$, where 


$$
\begin{aligned}
\xi(r) & :=\sum_{m=1}^{r} \frac{1-p_{\mathrm{fa}}}{\left(1-p_{\mathrm{fa}}\right) \frac{m}{r}+p_{\mathrm{md}}\left(1-\frac{m}{r}\right)}\left(\begin{array}{l}
r \\
m
\end{array}\right) \frac{1}{2^{r}}, \\
\gamma(r) & =\sum_{m=0}^{r-1} r \frac{p_{e}}{p_{\mathrm{md}}}\left(\begin{array}{l}
r \\
m
\end{array}\right) \frac{1}{2^{r}} .
\end{aligned}
$$

The proof is given in Appendix B.

One can similarly consider the dynamics of the Shannon capacity of the primary link at time $t$. The Shannon capacity of the primary link in band $b_{i_{\ell}}$ is given by:

$$
C^{\left(i_{\ell}\right)}(t):=\log _{2}\left(1+\frac{P_{p}}{\mathscr{N}_{0} W_{i_{\ell}}+d^{\eta} I^{\left(i_{\ell}\right)}(t)}\right)
$$

where $\mathscr{N}_{0} W_{i_{\ell}}$ is the total noise power in band $b_{i_{\ell}}, d$ is the distance between the primary transmitter and receiver, and $\eta$ is the path loss exponent. Now, by Proposition 4.2, as $N \rightarrow \infty$, the interference converges to its mean value almost surely. Thus, by the smoothness of the $\log _{2}(\cdot)$ function, we have the following proposition regarding the Shannon capacity of band $b_{i_{\ell}}$ (parallel to Proposition 4.2):

Proposition 4.4: $\quad$ Let $C^{\left(i_{\ell}\right)}(t)$ be the Shannon capacity of the primary link in band $b_{i_{\ell}}$ at time $t \in\left[t_{\ell}, t_{\ell+1}\right]$. Then, $C^{\left(i_{\ell}\right)}(t)$ converges to its mean value given by

$$
\begin{aligned}
& C^{\left(i_{\ell}\right)}(t) \rightarrow \\
& \log _{2}\left(1+\frac{P_{p} / d^{\eta}}{\mathscr{N}_{0} W_{i_{\ell}}+\frac{1}{r} I_{g}+\left(\alpha_{\ell} e^{-\left(t-t_{\ell}\right) / \tau_{s}}+\alpha p_{e}\left(1-e^{-\left(t-t_{\ell}\right) / \tau_{s}}\right)\right) I_{e}}\right)
\end{aligned}
$$

almost surely as $N \rightarrow \infty$.

Similarly, a general expression for the Shannon capacity of the primary link at any time $t$ (parallel to Eq. (14)), and the ensemble average of the Shannon capacity with respect to the primary link activity (parallel to Proposition 4.3) can be obtained. But, the details are omitted for brevity.

\subsection{Secondary Dynamics}

Recall that $\mathcal{I} \subseteq\{1,2, \cdots, N\}$ denotes the indices corresponding to the secondary users within a distance $R_{0}$ from the primary receiver. As before, let $t_{1}, t_{2}, \cdots$ denote the time instances corresponding to the state transitions of the primary link, and let $\mathcal{J}_{\ell}(t):=\left\{b_{i} \mid A^{(i)}\left(t_{\ell}^{+}\right)=1\right\}$, be the set of frequency bands in which the primary link is active after a transition at time $t_{\ell}$. Also, let $\mathcal{H}_{i}(t) \subseteq\{1,2, \cdots, r\}$ be the set of frequency bands across which user $c_{i}$ performs the GADIA algorithm, at time $t$. Note that for $i \notin \mathcal{I}$, we simply have $\mathcal{H}_{i}(t)=\{1,2, \cdots, r\}$. However, for $i \in \mathcal{I}, \mathcal{H}_{i}(t)$ includes all the $r$ frequency bands, except for those of which secondary user $c_{i}$ has knowledge of the activity of the primary link, and in which the primary link is active at time $t$. In this regard, $\mathcal{H}_{i}(t)$, for $i \in \mathcal{I}$ is not necessarily the same as $\mathcal{J}_{\ell}(t)$, unless the secondary users have genie knowledge of the activity of the primary link. Moreover, let $\gamma_{k}$, for $k=1,2, \cdots, N$ denote the channel between the secondary user $c_{k}$ and the primary transmitter. The network utility of the secondary users, $U_{s}(t)$, can be decomposed as:

$$
U_{s}(t)=U_{s s}(t)+U_{p s}(t)
$$

where $U_{s s}(t)$ and $U_{p s}(t)$ denote the secondary-to-secondary and primary-to-secondary interference experienced by the secondary users, respectively, and are given by:

$$
U_{s s}(t)=-\sum_{\substack{k, l=1 \\ k \notin \mathcal{I}, k \neq l}}^{N} J_{k l} \delta\left(s_{k}(t), s_{l}(t)\right),
$$

and

$$
U_{p s}(t)=-\sum_{\substack{k=1 \\ k \notin \mathcal{I}}}^{N} \sum_{j \in \mathcal{J}_{\ell}(t)} \delta\left(s_{k}(t), j\right) P_{k} P_{p} \gamma_{k},
$$

for $t \in\left[t_{\ell}, t_{\ell+1}\right]$, where $J_{k l}:=P_{k} P_{l} \alpha_{k l}$. Note that in the above summation we enforce the implicit assumption that if for some $i, \mathcal{H}_{i}(t)=\emptyset$, then $\delta\left(s_{i}(t), m\right)=0$ for any $m \in$ $\{1,2, \cdots, r\}$. Similar to the approach taken in [1], for $t \in$ $\left[t_{\ell}, t_{\ell+1}\right]$ we have:

$$
\frac{d}{d t}\left\langle U_{s s}(t)\right\rangle=-\frac{2}{\tau_{s}}\left(\left\langle U_{s s}(t)\right\rangle-\left\langle U_{s s}^{0}(t)\right\rangle\right),
$$

where $U_{s s}^{0}(t)$ is given by

$$
\begin{aligned}
& U_{s s}^{0}(t):=-\lim _{\beta \rightarrow \infty} \sum_{\substack{k, l=1 \\
k \neq l}}^{N} J_{k l} \mathbb{I}\left\{s_{l}(t) \in \mathcal{H}_{k}(t)\right\} \\
& \times \frac{\exp \left(-\beta\left(\sum_{j=1, j \neq k}^{N} J_{k j} \delta\left(s_{l}(t), s_{j}(t)\right)+\mathbb{I}\left\{s_{l}(t) \in \mathcal{J}_{\ell}(t)\right\} P_{k} P_{p} \gamma_{k}\right)\right)}{\sum_{m \in \mathcal{H}_{k}(t)} \exp \left(-\beta\left(\sum_{j=1, j \neq k}^{N} J_{k j} \delta\left(m, s_{j}(t)\right)+\mathbb{I}\left\{m \in \mathcal{J}_{\ell}(t)\right\} P_{k} P_{p} \gamma_{k}\right)\right)}
\end{aligned}
$$

with $\mathbb{I}\{\cdot\}$ denoting the logical indicator function. The indicator function is included to restrict the corresponding summation over $\sigma_{k}$ in the trace operator, to the index set $\mathcal{H}_{k}(t)$, for all $k=1,2, \cdots, N$.

Similarly, the dynamics of $\left\langle U_{p s}(t)\right\rangle$ is given by

$$
\frac{d}{d t}\left\langle U_{p s}(t)\right\rangle=-\frac{1}{\tau_{s}}\left(\left\langle U_{p s}(t)\right\rangle-\left\langle U_{p s}^{0}(t)\right\rangle\right),
$$

where $U_{p s}^{0}(t)$ is defined as

$$
\begin{aligned}
& U_{p s}^{0}(t):=-\lim _{\beta \rightarrow \infty} \sum_{k=1}^{N} \sum_{j \in \mathcal{J}_{\ell}} P_{k} P_{p} \gamma_{k} \mathbb{I}\left\{j \in \mathcal{H}_{k}(t)\right\} \\
& \times \frac{\exp \left(-\beta\left(\sum_{v=1, v \neq k}^{N} J_{k v} \delta\left(j, s_{v}(t)\right)+\mathbb{I}\left\{j \in \mathcal{J}_{\ell}(t)\right\} P_{k} P_{p} \gamma_{k}\right)\right)}{\sum_{m \in \mathcal{H}_{k}(t)} \exp \left(-\beta\left(\sum_{v=1, v \neq k}^{N} J_{k v} \delta\left(m, s_{v}(t)\right)+\mathbb{I}\left\{m \in \mathcal{J}_{\ell}(t)\right\} P_{k} P_{p} \gamma_{k}\right)\right)} .
\end{aligned}
$$


The details of the derivation of $U_{s s}^{0}(t)$ and $U_{p s}^{0}(t)$ are similar to that of $U_{0}(t)$ in [1], and are left to the reader. Note that the linear drift terms of the above differential equations have different time constants. It is possible to obtain nearequilibrium approximations to the above equations as in [1], given $\tau_{s} \ll \tau_{p}$, so that the interval $\left[t_{\ell}, t_{\ell+1}\right]$ is typically long enough for the above equations to reach near the equilibrium point. Also, note that for $t \gg \tau_{s}$, we have $\mathcal{H}_{i}(t)=\mathcal{J}_{\ell}(t)$ for all $i \in \mathcal{I}$, and $\mathcal{H}_{i}(t)=\{1,2, \cdots, r\}$ for all $i \notin \mathcal{I}$. The details of the near-equilibrium linearization are omitted for brevity.

\section{Discussion of the Main Results}

Theorem 4.1 studies the time evolution of the average number of active secondary users in a frequency band just occupied by the primary link, following a transition. In fact, for $N$ large enough, the theorem implies that the temporal evolution of the number of active secondary users is given by an exponential with time constant $\tau_{s}$. This is due to the fact that the normalized variance of the number of active users vanishes as $N \rightarrow \infty$.

Proposition 4.2 which is based on Theorem 4.1, gives a closed form expression for the interference experienced by the primary receiver in a frequency band which is just occupied following a state transition, asymptotically as $N \rightarrow$ $\infty$. In parallel, Proposition 4.4 gives a similar expression for the Shannon capacity of the primary link.

Proposition 4.3 concerns the average steady state behavior of the overall interference experienced by the primary link. The average interference experienced by the primary receiver is given in closed form based on the system parameters $R_{\min }, R_{0}, R_{\max }, \Lambda, r, \tau_{s}, \tau_{p}, p_{\mathrm{fa}}$, and $p_{\mathrm{md}}$. The result of Proposition 4.3 reveals the effect of the system parameters on the average interference experienced by the primary receiver. In particular, the term $\frac{1}{2} I_{g}$ reflects the average normalized interference from the secondary users beyond the radius $R_{0}$, which do not regulate their transmission based on the activity of the primary link. Hence, this portion of the average normalized interference can be considered as the "background" interference. The next term involving $I_{e}$, reflects the excess interference due to the delay and detection error of the secondary users in abiding by the vertical spectrum sharing regulations. This term can be made arbitrarily small if the miss detection error $p_{\mathrm{md}}$ and the ratio $\tau_{s} / \tau_{p}$ are small enough.

Finally, the dynamics of the network utility of the secondary users is explored in Sect. 4.2. Due to the existence of the primary link, the network utility of the secondary users is decomposed into two parts corresponding to the secondary-to-secondary and primary-to-secondary interference, respectively. It has been shown that the secondary-tosecondary and primary-to-secondary interference have linear drift terms with time constants of $\tau_{s} / 2$ and $\tau_{s}$, respectively.

\section{Simulation Results}

\subsection{Simulation Settings}

As for the simulations, we conform to the system model in Fig. 1. For this purpose, we normalize the distances as $R_{\min }=1 R_{0}=1.8$ and $R_{\max }=3$. Also, the distance between the primary receiver and transmitter is assumed to be $R_{\min }$, which corresponds to the worst case. We consider $100 \mathrm{sec}$ ondary users distributed uniformly between the two discs of radii $R_{\min }$ and $R_{\max }$. Also, we normalize the powers as $P_{s}=1$ and $P_{p}=500$. We consider two frequency bands available for transmission $(r=2)$. Finally, the time is normalized so that $\tau_{p}=2$ (recall that $1 / \tau_{p}$ is the transition rate of the activity state of the primary link). All the following simulations are performed for a fixed spatial distribution of the 100 secondary users, where 38 secondary users are located within the radius of $R_{0}$ from the primary receiver.

\subsection{Dynamics of $n^{(1)}(t), I(t)$ and $C_{p}(t)$}

In the first experiment, we consider the scenario where the primary transmitter is inactive in both bands from time $t=0$ to $t=0.2$. At time $t=0.2$, the primary link becomes active in band $b_{1}$. Also, we suppose that at this time, all the 100 users are in band $b_{1}$, and that $p_{\mathrm{md}}=p_{\mathrm{fa}}=0$.

Figure 2 shows the time evolution of $n^{(1)}(t), I(t)$ and $C_{p}(t)$ in this scenario, with $\tau_{1}=0.1$. As it can be observed from Fig. 2, the dynamics of these parameters perfectly matches the analytical results of Theorem 4.1, Proposition 4.2 and 4.4. That is, the values of $n^{(1)}(t), I(t)$ and $C_{p}(t)$ converge to their mean for all $t$, for $N$ large enough. Moreover, the interference and Shannon capacity of the primary receiver converge to their optimal values exponentially fast in $1 / \tau_{s}$, as predicted by the analytical results.

\subsection{The Effect of $\tau_{s}, p_{\mathrm{md}}$ and $p_{\mathrm{fa}}$ on the Dynamics}

In the second experiment, we consider the steady state of
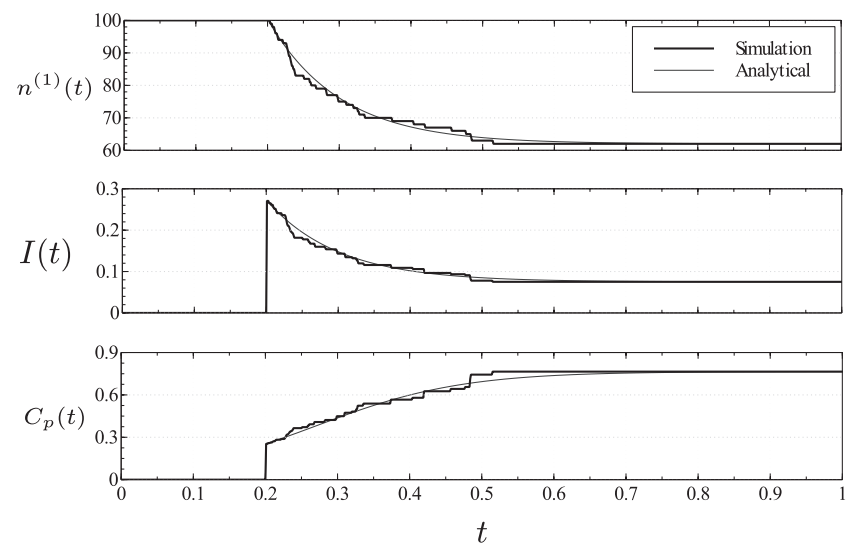

Fig. 2 Simulation vs. analytical results for the primary link interference, capacity and number of active secondary users. 


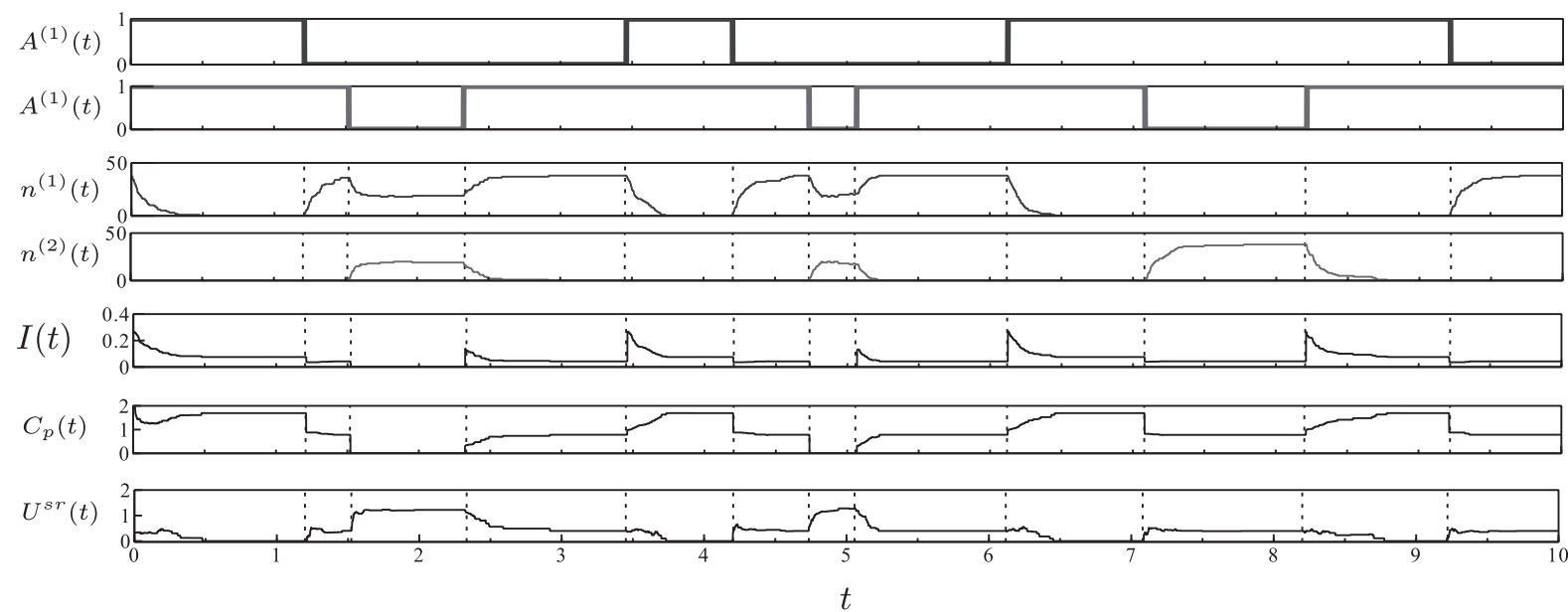

Fig. 3 System parameters vs. $t$, for $p_{\mathrm{md}}=p_{\mathrm{fa}}=0, \tau_{s}=0.1$, and $\tau_{p}=2$.

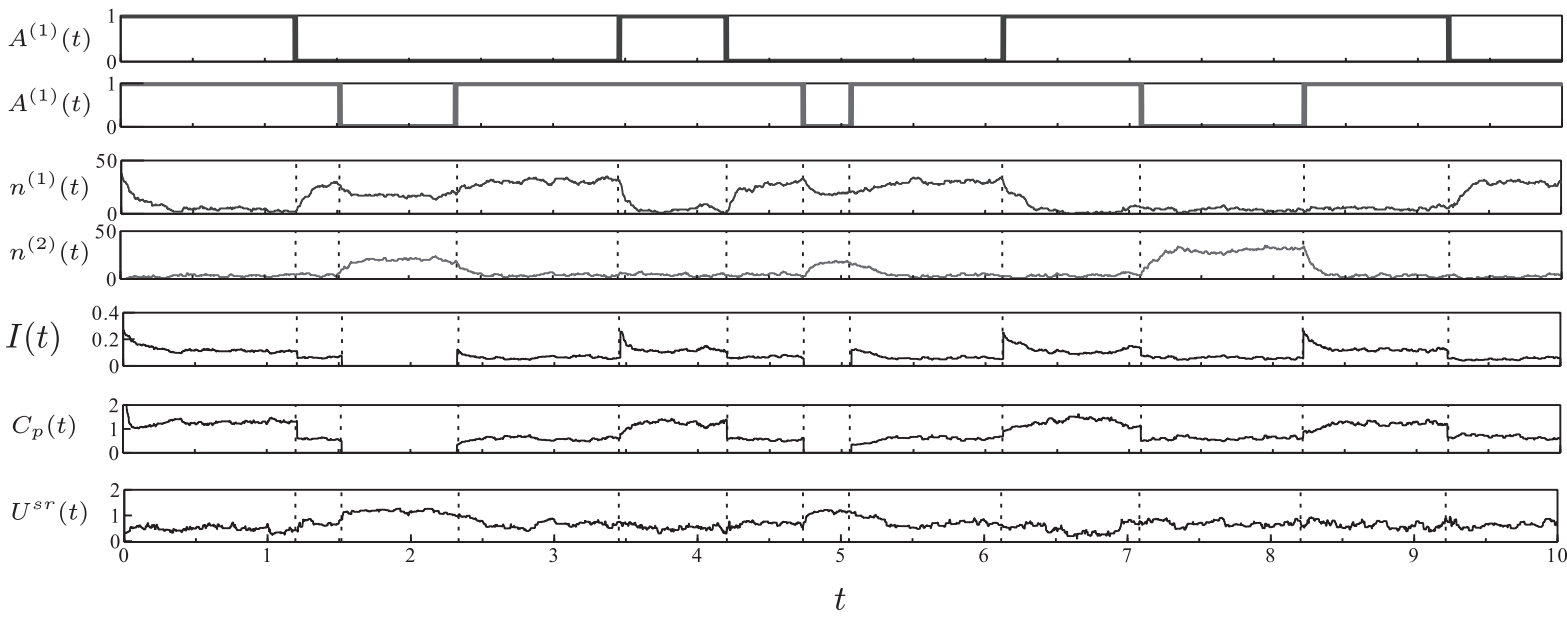

Fig. 4 System parameters vs. $t$, for $p_{\mathrm{md}}=p_{\mathrm{fa}}=0.1, \tau_{s}=0.1$, and $\tau_{p}=2$.

the system, where the activity of the primary link is modeled by a two state symmetric Markov model with transition rate $1 / \tau_{p}=0.5$. We record the number of active secondary users in each band, primary link interference and Shannon capacity, and the sum-rate of secondary users, $U^{s r}(t)$, for an interval of $10 \mathrm{~s}$.

Figure 3 shows the time evolution of the above quantities for $p_{\mathrm{md}}=p_{\mathrm{fa}}=0, \tau_{s}=0.1$ and $\tau_{p}=2$. Note that the GADIA algorithm is performed by the secondary users with a rate of $1 / \tau_{s}=10$ per user. As it can be observed from Fig. 3, in this case the optimal rate of the primary is not achieved instantaneously. Moreover, the sum-rate of the secondary users increases as the primary link becomes inactive in each of the frequency bands.

Finally, Fig. 4 shows the same quantities for $p_{\mathrm{md}}=$ $p_{\mathrm{fa}}=0.1$, and $\tau_{s}=0.1$ and $\tau_{p}=2$. In this case, the secondary users detect the activity state of the primary link with nonzero error probability. Hence, as it can be observed from Fig. 4, the primary interference and capacity, number of active users and the secondary sum-rate fluctuate around their optimal values, as predicted by Proposition 4.2.

\section{Conclusion}

We have studied the problem of spectrum sharing in cognitive radios in a vertical spectrum sharing scenario. In this scenario, the secondary users detect the activity of the primary links and regulate their transmissions accordingly, in order to avoid interfering with the primary users. Moreover, the secondary users share the spectrum using the Greedy Asynchronous Distributed Interference Avoidance (GADIA) algorithm while controlling their interference on the primary users. Several analytical results presented in this paper establish the temporal behavior of the system parameters such as primary interference and Shannon capacity and the aggregate interference of the secondary users. In particular, these results imply that the primary user achieves nearoptimal rates at all times, and the secondary users achieve near-optimal sum-rates by performing the GADIA algorithm. Finally, simulation studies reveal the validity of our analytical results. 


\section{References}

[1] B. Babadi and V. Tarokh, "GADIA: A greedy asynchronous distributed interference avoidance algorithm," IEEE Trans. Inf. Theory, vol.56, no.12, pp.6228-6252, Dec. 2010.

[2] B. Babadi and V. Tarokh, "Vertical spectrum sharing via distributed asynchronous interference avoidance in cognitive radio networks," Proc. IEEE Wireless Consumer Communication and Networking Conference (CCNC'12), Las Vegas, NV, Jan. 2012.

[3] P.K. Biswas and S. Phoha, "Self-organizing sensor networks for integrated target surveillance," IEEE Trans. Comput., vol.55, no.8, pp.1033-1047, Aug. 2006.

[4] R. Cendrillon, J. Huang, M. Chiang, and M. Moonen, "Autonomous spectrum balancing for digital subscriber lines," IEEE Trans. Signal Process., vol.55, no.8, pp.4241-4257, Aug. 2007.

[5] R. Etkin, A. Parekh, and D. Tse, "Spectrum sharing for unlicensed bands," IEEE J. Sel. Areas Commun., vol.25, no.3, pp.517-528, April 2007.

[6] S. Haykin, "Cognitive radio: Brain-empowered wireless communication,” IEEE J. Sel. Areas Commun., vol.23, no.2, pp.201-220, 2005.

[7] J. Huang, R.A. Berry, and M.L. Honig, "Distributed interference compensation for wireless networks," IEEE J. Sel. Areas Commun. vol.24, no.5, pp.1074-1084, May 2006.

[8] Federal Communications Commission, Second Report and Order in the matter of Unlicensed Operation in the TV Broadcast Bands, and Additional Spectrum for Unlicensed Devices Below $900 \mathrm{MHz}$ and in the $3 \mathrm{GHz}$ Band, FCC 08-260, 14 Nov. 2008, Available at: http://www.fcc.gov/Daily_Releases/Daily_Business/2008/ db1117/FCC-08-260A1.pdf

[9] W. Feller, Probability Theory, vol.I, John Wiley, New York, 1957 and vol.II, 1966.

[10] K. Harrison, M. Mishra, and A. Sahai, "How much white-space capacity is there?," Proc. 2010 IEEE Symposium on Dynamic Spectrum Access Networks, April 2010.

[11] C. Lacatus and D.C. Popescu, "Adaptive Interference Avoidance for Dynamic Wireless Systems: A Game-Theoretic Approach,” IEEE J. Sel. Top. Signal Process., vol.1, no.1, pp.189-202, June 2007.

[12] J. Neel, Analysis and design of cognitive radio networks and distributed radio resource management algorithms, Ph.D. dissertation, Dept. Electr. Comput. Eng., Virginia Polytechnic Inst. State Univ., Blacksburg, VA, 2006.

[13] J. Neel and J. Reed, "Performance of distributed dynamic frequency selection schemes for interference reducing networks," Milcom 2006, Washington D.C., Oct. 2006

[14] N. Nie and C. Comaniciu, "Adaptive channel allocation spectrum etiquette for cognitive radio networks," ACM MONET (Mobile Networks and Applications), special issue on "Reconfigurable radio technologies in support of ubiquitous seamless computing," 2006.

[15] A. Ozgur, O. Leveque, and D. Tse, "Hierarchical cooperation achieves optimal capacity scaling in ad hoc networks," IEEE Trans. Inf. Theory, vol 53, no.10, pp.3549-3572, Oct. 2007.

[16] A. Papoulis, Probability, Random Variables, and Stochastic Processes, 3rd ed. McGraw-Hill, 1991.

[17] D.C. Popescu and C. Rose, Interference Avoidance Methods for Wireless Systems, Kluwer Academic Publishing, 2004.

[18] C. Rose, S. Uluks, and R. Yates, "Wireless systems and interference avoidance," IEEE Trans. Wirel. Commun., vol.1, no.3, pp.415-428, July 2002.

[19] A. Sahai, R. Tandra, and M. Mishra, "Spectrum sensing: Fundamental limits," in Cognitive Radios: System Design Perspective, ed R.W. Brodersen and D. Cabric, Springer, 2009.

[20] R. Tandra, M. Mishra, and A. Sahai, "What is a spectrum hole and what does it take to recognize one?," Proc. IEEE Special Issue on Cognitive Radio, pp.824-848, May 2009.

[21] M. Vu and V. Tarokh, "Scaling laws of single-hop cognitive net- works," IEEE Trans. Wireless Commun., vol.8, no.8, pp.4089-4097, Aug. 2009.

[22] Y. Xing, R. Chandramouli, S. Mangold, and S. Shankar, "Dynamic spectrum access in open spectrum wireless networks," IEEE J. Sel. Areas Commun., vol.24, no.3, pp.626-637, March 2006.

[23] W. Yu, G. Ginis, and J.M. Cioffi, "Distributed multiuser power control for digital subscriber lines," IEEE J. Sel. Areas Commun., vol.20, no.5, pp.1105-1115, June 2002.

\section{Appendix A: $\quad$ Proof of Theorem 4.1}

Recall the Itô equation [9] given by Eq. (10):

$$
d a_{k}^{\left(i_{\ell}\right)}(t)=-a_{k}^{\left(i_{e}\right)}(t) d N_{k}+\left(1-a_{k}^{\left(i_{e}\right)}(t)\right) d N_{k}^{\prime}
$$

Taking the expectation of both sides yields:

$$
d \mathcal{E}_{s}\left\{a_{k}^{\left(i_{e}\right)}(t)\right\}=-\frac{1}{\tau_{s}} \mathcal{E}\left\{a_{k}^{\left(i_{e}\right)}(t)\right\} d t+\frac{p_{e}}{\tau_{s}} d t
$$

Therefore,

$$
\mathcal{E}_{s}\left\{a_{k}^{\left(i_{\ell}\right)}(t)\right\}=a_{k}^{\left(i_{\ell}\right)}\left(t_{\ell}\right) e^{-\left(t-t_{\ell}\right) / \tau_{s}}+p_{e}\left(1-e^{-\left(t-t_{\ell}\right) / \tau_{s}}\right)
$$

and

$$
\mathcal{E}_{s}\left\{n^{\left(i_{\ell}\right)}(t)\right\}=n_{\ell} e^{-\left(t-t_{\ell}\right) / \tau_{s}}+\alpha N p_{e}\left(1-e^{-\left(t-t_{\ell}\right) / \tau_{s}}\right)
$$

One can similarly compute the variance of $a_{k}^{\left(i_{t}\right)}(t)$, hence $n^{\left(i_{\ell}\right)}(t)$. By the Itô rule, we have:

$$
\begin{aligned}
d\left(a_{k}^{\left(i_{\ell}\right)}(t)^{2}\right)= & \left\{\left(a_{k}^{\left(i_{\ell}\right)}(t)-a_{k}^{\left(i_{\ell}\right)}(t)\right)^{2}-a_{k}^{\left(i_{\ell}\right)}(t)^{2}\right\} d N_{k} \\
& +\left\{\left(a_{k}^{\left(i_{\ell}\right)}(t)+1-a_{k}^{\left(i_{e}\right)}(t)\right)^{2}-a_{k}^{\left(i_{\ell}\right)}(t)^{2}\right\} d N_{k}^{\prime} \\
= & -a_{k}^{\left(i_{\ell}\right)}(t)^{2} d N_{k}+\left(1-a_{k}^{\left(i_{\ell}\right)}(t)^{2}\right) d N_{k}^{\prime}
\end{aligned}
$$

Therefore,

$$
\mathcal{E}_{s}\left\{a_{k}^{\left(i_{\ell}\right)}(t)^{2}\right\}=a_{k}^{\left(i_{\ell}\right)}\left(t_{\ell}\right)^{2} e^{-t / \tau_{s}}+p_{e}\left(1-e^{-t / \tau_{s}}\right) .
$$

We then have:

$$
\begin{aligned}
\mathcal{E}_{s}\left\{n^{\left(i_{\ell}\right)}(t)^{2}\right\} & =\mathcal{E}_{s}\left\{\left(\sum_{k} a_{k}^{\left(i_{\ell}\right)}(t)\right)^{2}\right\} \\
& =\sum_{k} \mathcal{E}_{s}\left\{a_{k}^{\left(i_{\ell}\right)}(t)^{2}\right\}+\sum_{\substack{k, m \\
k \neq m}} \mathcal{E}_{s}\left\{a_{k}^{\left(i_{\ell}\right)}(t)\right\} \mathcal{E}_{s}\left\{a_{m}^{\left(i_{\ell}\right)}(t)\right\},
\end{aligned}
$$

which can be simplified as:

$$
\begin{aligned}
\mathcal{E}_{s}\left\{n^{\left(i_{\ell}\right)}(t)^{2}\right\}=n_{\ell} e^{-\left(t-t_{\ell}\right) / \tau_{s}}+\alpha N p_{e}\left(1-e^{-\left(t-t_{\ell}\right) / \tau_{s}}\right) \\
+n_{\ell}\left(n_{\ell}-1\right)\left(e^{-\left(t-t_{\ell}\right) / \tau_{s}}+p_{e}\left(1-e^{-\left(t-t_{\ell}\right) / \tau_{s}}\right)\right)^{2} \\
+\left(\alpha N-n_{\ell}\right)\left(\alpha N-n_{\ell}-1\right) p_{e}^{2}\left(1-e^{-\left(t-t_{\ell}\right) / \tau_{s}}\right)^{2} \\
+2\left(\alpha N-n_{\ell}\right) n_{\ell} p_{e}\left(1-e^{-\left(t-t_{\ell}\right) / \tau_{s}}\right)\left(e^{-\left(t-t_{\ell}\right) / \tau_{s}}+p_{e}\left(1-e^{-\left(t-t_{\ell}\right) / \tau_{s}}\right)\right) \\
=\left(n_{\ell} e^{-\left(t-t_{\ell}\right) / \tau_{s}}+\alpha N p_{e}\left(1-e^{-\left(t-t_{\ell}\right) / \tau_{s}}\right)\right)^{2}+ \\
\quad\left(1-e^{-\left(t-t_{\ell}\right) / \tau_{s}}\right) \times \\
\quad \quad\left\{n_{\ell}\left(1-2 p_{e}\right) e^{-\left(t-t_{\ell}\right) / \tau_{s}}+\alpha N p_{e}\left(1-p_{e}\left(1-e^{-\left(t-t_{\ell}\right) / \tau_{s}}\right)\right)\right\} .
\end{aligned}
$$


Therefore, the normalized variance of $n^{\left(i_{\ell}\right)}(t)$ is given by:

$$
\begin{aligned}
\sigma_{n^{\left(i_{\ell}\right)}(t)}^{2}:= & \frac{1}{N^{2}} \mathcal{E}_{s}\left\{n^{\left(i_{\ell}\right)}(t)^{2}-\mathcal{E}_{s}\left\{n^{\left(i_{\ell}\right)}(t)\right\}^{2}\right\} \\
= & \frac{\left(1-e^{-\left(t-t_{\ell}\right) / \tau_{s}}\right)}{N} \\
& \times\left\{\alpha_{\ell}\left(1-2 p_{e}\right) e^{-\left(t-t_{\ell}\right) / \tau_{s}}+\alpha p_{e}\left(1-p_{e}+p_{e} e^{-\left(t-t_{\ell}\right) / \tau_{s}}\right)\right\},
\end{aligned}
$$

which establishes the claim of the theorem.

\section{Appendix B: $\quad$ Proof of Proposition 4.3}

In order to compute the ensemble average with respect to the $r$ independent Markov processes, we invoke the ergodicity of the underlying Poisson processes [16] and carry out the averaging in the time domain. Consider the frequency band $b_{j}$, and the corresponding transition times of type 1 and 2 , given by $\left\{t_{k}^{(j)}\right\}_{k=1}^{\ell}$ and $\left\{t_{k}^{\prime(j)}\right\}_{k=1}^{\ell}$, respectively, with the interlacing property given in Eq. (13). The temporal average of the inner summation in the definition of $\tilde{I}_{e}(t)$, in band $b_{j}$ in the time interval $\left[t_{1}^{(j)}, t_{\ell}^{\prime(j)}\right]$ is given by Eq. (A. 4).

$$
\begin{aligned}
& \int_{0}^{t^{\prime}{ }^{(j)}} \sum_{k=1}^{\ell}\left\{\alpha_{k}^{(j)} e^{-\left(t-t_{k}^{(j)}\right) / \tau_{s}}+\alpha p_{e}\left(1-e^{-\left(t-t_{k}^{(j)}\right) / \tau_{s}}\right)\right\} \\
& \lim _{\ell \rightarrow \infty} \frac{\left(u\left(t-t_{k}^{(j)}\right)-u\left(t-{t^{\prime}}_{k}^{(j)}\right)\right) I_{e} d t}{\left(t_{\ell}^{\prime(j)}-t_{1}^{(j)}\right)}
\end{aligned}
$$

Dividing the numerator and denominator of Eq. (A.4) by $2 \ell-1$ (the number of intervals between the transitions) and simplifying the integral in the numerator results in Eq. (A. 5).

$$
\lim _{\ell \rightarrow \infty} \frac{\frac{1}{2 \ell-1} \sum_{k=1}^{\ell} \int_{t_{k}^{(j)}}^{t^{\prime(j)}}\left\{\alpha_{k}^{(j)} e^{-\left(t-t_{k}^{(j)}\right) / \tau_{s}}+\alpha p_{e}\left(1-e^{-\left(t-t_{k}^{(j)}\right) / \tau_{s}}\right)\right\} I_{e} d t}{\frac{1}{2 \ell-1} \sum_{k=1}^{\ell}\left(t_{k}^{\prime(j)}-t_{k}^{(j)}\right)+\frac{1}{2 \ell-1} \sum_{k=1}^{\ell-1}\left(t_{k+1}^{(j)}-t_{k}^{(j)}\right)}
$$

Next, evaluating the integral yields Eq. (A. 6).

$$
\frac{\lim _{\ell \rightarrow \infty} \frac{1}{2 \ell-1} \sum_{k=1}^{\ell}\left\{\alpha_{k}^{(j)} \tau_{s}\left(1-e^{-\left(t^{(j)}{ }_{k}-t_{k}^{(j)}\right) / \tau_{s}}\right)+\alpha p_{e}\left(\left(t^{\prime}{ }_{k}^{(j)}-t_{k}^{(j)}\right)-\tau_{s}\left(1-e^{-\left(t^{\prime}{ }_{k}^{(j)} t_{k}^{(j)}\right) / \tau_{s}}\right)\right)\right\} I_{e}}{\lim _{\ell \rightarrow \infty} \frac{1}{2 \ell-1} \sum_{k=1}^{\ell}\left(t^{\prime}{ }_{k}^{(j)}-t_{k}^{(j)}\right)+\lim _{\ell \rightarrow \infty} \frac{1}{2 \ell-1} \sum_{k=1}^{\ell-1}\left(t_{k+1}^{(j)}-t^{\prime(j)}\right)}
$$

Now, consider the denominator of Eq. (A. 6). Since the points $t_{k}^{(j)}$ and $t_{k}^{(j)}$ are samples from a Poisson point process, the intervals $\left(t_{k}^{\prime(j)}-t_{k}^{(j)}\right)$ and $\left(t_{k+1}^{(j)}-t_{k}^{\prime(j)}\right)$ are samples from an exponential distribution. That is,

$$
p_{t_{k}^{(j)}}\left(t \mid t_{k}^{(j)}\right)=p_{t_{k+1}^{(j)}}\left(t \mid t_{k}^{(j)}\right)=\frac{1}{\tau_{p}} e^{-t / \tau_{p}}
$$

Also, note that $\alpha_{k}^{(j)}$ is statistically independent from $\left(t^{\prime(j)}-\right.$ $\left.t_{k}^{(j)}\right)$ and $\left(t_{k+1}^{(j)}-t_{k}^{(j)}\right)$, since $\alpha_{k}^{(j)}$ is only a function of $t_{k}^{(j)}$. Therefore by invoking the ergodicity of the underlying Poisson process, we have:

$$
\begin{aligned}
\lim _{\ell \rightarrow \infty} \frac{1}{2 \ell} \sum_{k=1}^{\ell}\left(t_{k}^{\prime(j)}-t_{k}^{(j)}\right) & =\lim _{\ell \rightarrow \infty} \frac{1}{2 \ell} \sum_{k=1}^{\ell-1}\left(t_{k+1}^{(j)}-t_{k}^{(j)}\right) \\
& =\frac{1}{2} \int_{0}^{\infty} \frac{t}{\tau_{p}} e^{-t / \tau_{p}} d t=\frac{1}{2} \tau_{p} .
\end{aligned}
$$

Similarly, taking the limit of $\ell \rightarrow \infty$ in the numerator and replacing $\alpha_{k}^{(j)}$ by $\mathcal{E}_{p}\left\{\alpha_{k}^{(j)}\right\}$, we get Eq. (A. 7).

$$
\frac{\int_{0}^{\infty}\left\{\mathcal{E}_{p}\left\{\alpha_{k}^{(j)}\right\}_{\tau_{s}}\left(1-e^{-t / \tau_{s}}\right)+\alpha \mathcal{E}_{p}\left\{p_{e}\right\}\left(t-\tau_{s}\left(1-e^{-t / \tau_{s}}\right)\right)\right\} \frac{I_{e}}{\tau_{p}} e^{-t / \tau_{p}} d t}{\frac{\tau_{p}}{2}+\frac{\tau_{p}}{2}}
$$

Recall that, $\alpha_{k}^{(j)}=n^{(j)}\left(t_{k}^{(j)}\right) / N$. Thus, we need to evaluate $\mathcal{E}_{p}\left\{n^{(j)}\left(t_{k}^{(j)}\right)\right\}$ and $\mathcal{E}_{p}\left\{p_{e}\right\}$ in the steady state. Assuming that $\tau_{s} \ll \tau_{p}$, we first show that

$$
\mathcal{E}_{p}\left\{n^{(j)}\left(t_{k}^{(j)}\right)\right\}=\frac{\xi(r)}{r} \Lambda \pi\left(R_{0}^{2}-R_{\min }^{2}\right),
$$

where $\xi(r)$ is as defined in the satement of Proposition 4.3. To see this, first note that $\Lambda \pi\left(R_{0}^{2}-R_{\min }^{2}\right)$ is the number of secondary users located between radii $R_{\min }$ and $R_{0}$. Suppose that at time $t_{k}^{(j)}$ the set of frequency bands $\mathcal{J}_{k}^{c}:=$ $\{1,2, \cdots, r\} \backslash \mathcal{J}_{k}$ are not occupied by the primary link, and let $1 \leqslant m:=\left|\mathcal{J}_{k}^{c}\right|$. Then, for $t \gg \tau_{s}$, let $p_{a}$ and $p_{a}^{\prime}$ be the probabilities that a secondary user, chosen at random, uses the frequency band $j \in \mathcal{J}_{k}^{c}$ or $j \notin \mathcal{J}_{k}^{c}$, respectively. Then, we must have $m p_{a}+(r-m) p_{a}^{\prime}=1$. Moreover, due to the detection error of the activity state of the primary link, we have $p_{a} / p_{a}^{\prime}=\left(1-p_{\mathrm{fa}}\right) / p_{\mathrm{md}}$. Solving the above equations for $p_{a}$ yields:

$$
p_{a}=\frac{1-p_{\mathrm{fa}}}{m\left(1-p_{\mathrm{fa}}\right)+(r-m) p_{\mathrm{md}}}
$$

Hence, there are $p_{a} \Lambda \pi\left(R_{0}^{2}-R_{\min }^{2}\right)$ users on average in each of the unoccupied bands, given $\left|\mathcal{J}_{k}^{c}\right|=m$. Finally, in the steady state, the probability of $\left|\mathcal{J}_{k}\right|=m$ is given by the Binomial density $\mathscr{B}\left(m ; \frac{1}{2}, r\right)$. Averaging over $m$, yields Eq. (A.8). Note that, the condition of $\tau_{s} \ll \tau_{p}$ implies that the secondary users have enough time to sense the spectrum and occupy the available frequency bands between any two consecutive state transitions of the primary. Similarly, by averaging over $m$, we get $\mathcal{E}_{p}\left\{p_{e}\right\}=\frac{\gamma(r)}{r} p_{\mathrm{md}}$. Evaluating the integral and simplifying Eq. (A.7) yields:

$$
\mathcal{E}_{p}\left\{\mathcal{E}_{s}\{I(t)\}\right\}=\frac{1}{2} I_{g}+\frac{1}{2} \alpha\left(\frac{\xi(r) \tau_{s}+\gamma(r) p_{\mathrm{md}} \tau_{p}}{\tau_{s}+\tau_{p}}\right) I_{e} .
$$

Finally, by Proposition 4.2,

$$
\mathcal{E}_{p}\{I(t)\} \rightarrow \mathcal{E}_{p}\left\{\mathcal{E}_{s}\{I(t)\}\right\}
$$

as $N \rightarrow \infty$, almost surely. This establishes the claim of the proposition. 


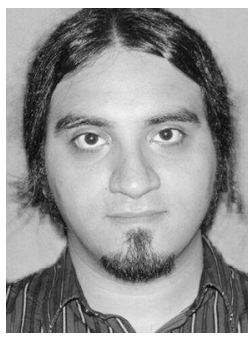

Behtash Babadi received the B.Sc. degree in electrical engineering from Sharif University of Technology, Tehran, Iran in 2006 and the M.Sc. and Ph.D. degrees in engineering sciences from Harvard University, Cambridge, MA, USA in 2008 and 2011, respectively. He is currently a post-doctoral fellow at the Department of Anesthesia, Critical Care and Pain Medicine, Massachusetts General Hospital, Boston, MA, USA and a research affiliate at the Department of Brain and Cognitive Sciences, Massachusetts Institute of Technology, Cambridge, MA, USA and the School of Engineering and Applied Sciences, Harvard University, Cambridge, MA, USA. His research interests include dynamic spectrum access networks, adaptive signal processing, information theory, compressed sensing and biological signal processing.

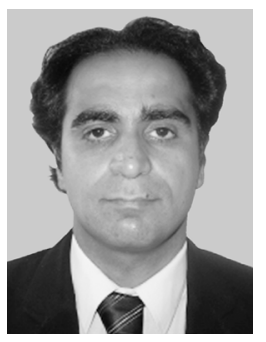

Vahid Tarokh received the M.Sc. degree from the University of Windsor, Windsor, ON, Canada, in 1992 and the Ph.D. degree in electrical engineering from the University of Waterloo, Waterloo, ON, Canada, in 1995. He worked at AT\&T Labs-Research until August 2000, where he was the head of the Department of Wireless Communications and Signal Processing. $\mathrm{He}$ then joined the Department of Electrical Engineering and Computer Sciences (EECS) at MIT as an associate professor. In 2002, he joined Harvard University as a professor and senior fellow. Dr. Tarokh's research interest is mainly focused in the areas of information theory, signal processing, communications and networking. He has received a number of awards, and holds 2 honorary degrees. 